\title{
Composition floristique, structure et menaces de la végétation de la ligne côtière de la Réserve de Faune de Douala-Edéa
}

\author{
Hyacinthe ANGONI ${ }^{*}$, Raymond S. ONGOLO ${ }^{1}$, Jean Baptiste NGODO MELINGUI ${ }^{1}$ et \\ Marie Laure NGO MPECK ${ }^{2}$ \\ ${ }^{1}$ Université de Yaoundé I, Faculté des Sciences, Département de BPV, BP: 812 Yaoundé, Cameroun. \\ ${ }^{2}$ Programme GEF/SGP/NUDP. \\ *Auteur correspondant ; E-mail: angonih@yahoo.fr, Tel:+23799194900
}

\begin{abstract}
RESUME
Une étude a été menée sur sa ligne côtière dans la partie Nord de la Réserve de Faune de Douala Edéa (RFDE) entre novembre 2011 et septembre 2012, dans le but de décrire la végétation des côtes sableuses et contribuer ainsi à la connaissance de la biodiversité de la Réserve de Douala Edéa. La méthodologie utilisée a consisté à la réalisation des inventaires botaniques basés sur la mise en place de parcelles de $50 \mathrm{~m}$ x $20 \mathrm{~m}(0,1$ ha) chacune à l'intérieur desquelles les individus ligneux ayant plus de $1 \mathrm{~cm}$ de DBH et les plantes herbacées sont recensés. Les échantillons en fleurs ou stériles ont été collectés et identifiés à l'herbier national de Yaoundé. Les menaces sur la biodiversité ont été également inventoriées en longeant les sections de plages identifiées. Les résultats montrent que les végétaux recensés se répartissent en 103 espèces, 89 genres et 56 familles. Avec un indice de Shannon de 3,56 bits, la diversité floristique de la ligne côtière de la RFDE reste faible. La structure en classes de diamètres de la végétation révèle un peuplement jeune, présentant une forte tendance à la régénération. Lophira alata et Terminalia catappa sont les espèces dominantes avec respectivement 40 et $16,5 \%$ du recouvrement total. Le phénomène d'érosion côtière, couplé et accentué par celui de l'élévation du niveau marin, constituent une menace à moyen et à long termes pour la biodiversité côtière de la réserve.
\end{abstract}

(C) 2018 International Formulae Group. All rights reserved.

Mots clés : Réserve de Faune de Douala-Edéa, ligne côtière, phytodiversité, structure floristique, érosion côtière.

\section{Floristic composition, structure and threats of the vegetation of the coastal line of the Douala-Edéa Wildlife Reserve}

\begin{abstract}
A study was conducted on its coastal line in the northern part of the Douala Edéa Wildlife Reserve (RFDE) between November 2011 and September 2012, in order to describe the vegetation of the sandy coasts and thus contribute to the knowledge of biodiversity of the Douala Edéa Reserve. The methodology used consisted of the realization of the botanical inventories based on the placement of parcels of $50 \mathrm{~m} \times 20 \mathrm{~m}(0.1$ ha) each within which the ligneous individuals having more than $1 \mathrm{~cm}$ of DBH and the herbaceous plants are identified. Samples in bloom or sterile were collected and identified at the Yaoundé National Herbarium. Threats to biodiversity have also been inventoried along the identified beach sections. The results show that the plants surveyed are divided into 103 species, 89 genera and 56 families. With a 3.56-bit Shannon index, the floristic diversity of the RFDE coastal line remains low. The structure in diameter classes of vegetation reveals a young stand, with a strong tendency to regeneration. Lophira alata and Terminalia catappa are the dominant species with respectively 40 and $16.5 \%$ of total recovery. The coastal erosion phenomenon, coupled and accentuated by sea level rise, poses a medium and long-term threat to the coastal biodiversity of the reserve. (c) 2018 International Formulae Group. All rights reserved.
\end{abstract}

Keywords: Douala-Edéa Wildlife Reserve, coastal line, phytodiversity, floristic structure, coastal erosion. 


\section{INTRODUCTION}

La zone côtière camerounaise est caractérisée par des écosystèmes variés et riches en biodiversité (De Kam et al., 2002). Cette zone, particulièrement sensible, voit sa biodiversité de plus en plus menacée. Les activités humaines dont la création de zones agro-industrielles, agricoles, la pêche et les prospections minières ont occasionné la dégradation progressive de l'environnement, la pollution du milieu côtier et la diminution des ressources biologiques de valeur (Nanko et al., 2010).

La Réserve de Faune de Douala-Edéa, ouverte sur l'océan atlantique, renferme divers écosystèmes dont la forêt, les lacs, les marécages, les mangroves et les cordons littoraux. Une faune abondante trouve ici un habitat privilégié dont certaines espèces présentent un intérêt particulier pour la conservation.C'est le cas de l'éléphant de forêt (Loxodonta africana cyclotis), le lamantin (Trichechus senegalensis), le chimpanzé (Pan troglodytes) et quatre espèces de tortues marines qui fréquentent les côtes de la réserve (Chelonia mydas, Lepidochelys olivacea, Dermochelys coriacea et Eretmochelys imbricata) (Angoni, 2011).

Le potentiel biologique de la réserve est encore à ce jour mal connu le long de la ligne côtière. Les études botaniques et ornithologiques ont été réalisées dans les mangroves tandis le potentiel floristique des cordons littoraux n'est pas connu. Pourtant un besoin de mettre en valeur cette aire protégée à travers une transformation en parc national se fait sentir (Anonyme, 2006).

La présente étude a pour objectif de décrire la végétation des plages et arrièreplages de la réserve. Spécifiquement, il s'agit de :

- déterminer la composition et la diversité floristiques de la végétation de la ligne côtière;

- déterminer la structure de la végétation des systèmes d'arrière-plages ;

- identifier des menaces qui pèsent sur la biodiversité de la ligne côtière de la réserve.

\section{MATERIEL ET METHODES \\ Site d'étude}

La Réserve de Faune de Douala-Edéa (RFDE), créée le 19 Novembre 1932, a une superficie d'environ 160000 ha et se localise dans le département de la Sanaga-maritime et dans le Département du Wouri, Région du Littoral. Elle est constituée de deux parties inégales : la plus grande, au Sud, se trouve entre les embouchures de la Sanaga au Nord et du Nyong au Sud ; l'autre partie s'étend le long de la côte Nord de la Sanaga jusqu'à la pointe de Souelaba et est limitée à l'Est par la crique de Kwa-Kwa.

L'étude se déroule le long des plages des villages Balondo (pointe de Souellaba) (une parcelle), Youmè I (une parcelle), Moukoukè et Kombo-Mukala (une parcelle), Mombo (une parcelle) et des inventaires de pollution le long des plages de Yoyo, soit au total près de 20 km de côtes (Figure 1).

\section{Méthodologie}

Cette étude consiste en un inventaire botanique des espèces herbacées et ligneuses le long des plages de la ligne côtière Nord de la réserve.

Les arbres ayant un diamètre à hauteur de poitrine $(\mathrm{DBH})$ au moins égal à $10 \mathrm{~cm}$ sont recensés. Les paramètres calculés sont les indices de diversité, la densité et la surface terrière.

Un inventaire des menaces directes qui pèsent sur la biodiversité de la ligne côtière sera fait par une identification d'indices de pollution et des destructions de la végétation en marchant le long des sections de plages.

\section{Dispositif expérimental}

Le dispositif d'inventaire botanique est basé sur la mise en place de parcelles rectangulaires. Celles-ci ont pour dimensions $50 \mathrm{~m}$ de long sur $20 \mathrm{~m}$ de large, soit une surface de $1000 \mathrm{~m}^{2}(0,1 \mathrm{ha})$ selon Angoni (2005), . Chaque parcelle est divisée en deux sous-parcelles de dimensions $50 \mathrm{~m}$ x $10 \mathrm{~m}$.

A l'intérieur de chaque sous parcelle sont installées trois placettes de $1 \mathrm{~m}$ x $1 \mathrm{~m}$ à l'intérieur desquelles s'effectue l'inventaire des plantes herbacées. Un indice d'abondance-dominance est noté pour chacune des espèces dans chaque parcelle (Braun-Blanquet, 1932).

L'inventaire a été réalisé dans cinq parcelles dans la partie Nord de la réserve. La surface totale du dispositif expérimental revient alors à $4000 \mathrm{~m}^{2}(0,4 \mathrm{ha})$ et $4 \mathrm{~m}^{2}$ pour l'étude des herbacées.

\section{Mesures biométriques}

Les circonférences des ligneux sont mesurées à l'aide d'un ruban métrique, à une hauteur de $1,30 \mathrm{~m}$ du sol tel que pratiqué par de nombreux auteurs (Vivien et Faure, 1985 ; Husch et al., 2003).

Le diamètre est obtenu par la relation suivante : Diamètre $=$ Circonférence $/ \pi$. 


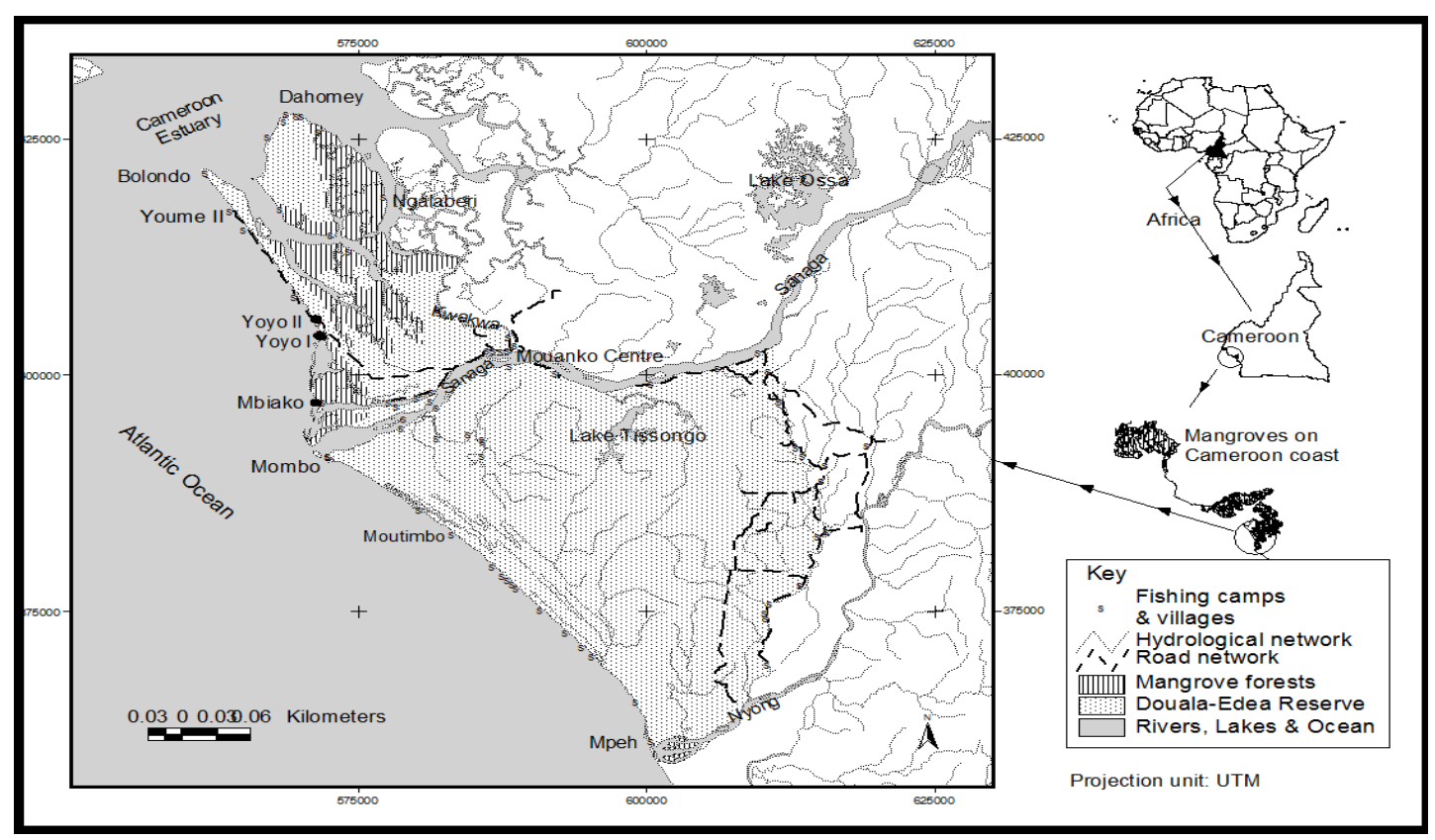

Figure 1 : Localisation de la Réserve de Faune de Douala-Edéa.

\section{Analyse des données}

La diversité floristique est évaluée à travers la richesse spécifique (RS), l'indice de Shannon (1948), l'indice d'équitabilité de Piélou (1965) et l'indice de Simpson (1949).

La structure floristique a été analysée par la détermination de la distribution des individus dans les classes de diamètre, de la densité (ou abondance) et de la surface terrière (dominance ou recouvrement). Ces variables écologiques permettent d'apprécier la dynamique du peuplement ligneux (Kimpouni et al., 2008).

\section{RESULTATS}

\section{Composition floristique}

Au total, 103 espèces dont 89 genres et 56 familles ont été recensés le long des côtes de la Réserve de Faune de Douala-Edéa (Tableau 1); 77 genres sont représentés par une seule espèce. C'est le cas des genres Albizia, Caesalpinia ou Hibiscus. De la même, 42 familles ne sont représentées que par un seul genre chacune. C'est le cas de la famille des Ixonanthaceae avec le genre
Ochtocosmus, ou des Araceae avec le genre Anchomanes.

\section{Diversité des familles}

Sept familles (Fabaceae, Rubiaceae, Convolvulaceae, Euphorbiaceae, Annonaceae, Arecaceae et Poaceae) englobent $40 \%$ du nombre total des espèces inventoriées (Tableau 2). Les familles les plus représentées en nombre d'espèces sont les Fabaceae et les Rubiaceae avec chacune $8,42 \%$ du total des espèces (Figure 2 ).

\section{Plages de sable}

Les familles les plus représentées sur les plages sableuses sont les Fabaceae et Rubiaceae avec respectivement 11 et 5 espèces. Les herbacées ayant les plus grands indices d'abondance-dominance sont Aframomum sp., Canavalia rosea, Vigna gracilis, Ipomoea mauritiana, I. pes-caprae.

Les arbres et arbustes les plus rencontrés sur les plages sont Calophyllum inophyllum, Dalbergia ecastaphyllum et Terminalia catappa. Notons également la présence de cocotiers (Cocos nucifera) sur tout le long des plages. 


\section{Végétation d'arrière-plage}

Les arrière-plages sont des milieux ouverts, souvent par l'action de l'homme.

Le sous-bois par conséquent est clair en fonction du degré d'ouverture de la canopée (Figure 3). Les herbacées les plus abondantes sont Nephrolepis biserrata, Aframomum sp., Costus tappenbeckianus, Manihot esculenta, Sarcophrynium brachystachium.

L'indice de Shannon, pour l'ensemble des parcelles, atteint la valeur de 3,56 bits. L'indice d'équitabilité de Piélou, qui exprime la régularité, la répartition équitable des individus au sein des espèces, est de 0,49. Enfin, l'indice d'hétérogénéité de Simpson atteint la valeur de 0,13. Ces résultats montrent qu'il y a $87 \%$ de probabilité que deux individus de ligneux choisis au hasard n'appartiennent pas à la même espèce.

\section{Structure de la végétation d'arrière-plage Répartition des individus par classes de diamètre}

La distribution des ligneux par classes de diamètre donne une image instantanée de la population d'arbres. La densité de cette dernière est de 938 pieds/ha. Cette distribution montre un très grand nombre de petits individus par rapport aux grands, et une réduction du nombre d'individus lorsqu'on passe d'une classe de diamètre à une autre (Figure 4). En effet, les individus de moins de $30 \mathrm{~cm}$ de diamètre regroupent plus de $94 \%$ des effectifs inventoriés. Le pourcentage élevé de la classe I témoigne d'une régénération naturelle installée.

\section{Abondance relative des taxons}

Les résultats présentés ici ne concernent que les individus de toutes classes de diamètre. Les familles les plus abondantes de la ligne côtière de la RFDE sont les Combretaceae avec 102,5 pieds/ha et plus de $27 \%$ des effectifs et les Loganiaceae (62,5 pieds/ha et $16,6 \%$ des effectifs). Etant donné qu'une famille n'est représentée que par une seule espèce, les espèces ayant les plus grandes abondances sont Terminalia catappa et Anthocleista schweinfurthii.

\section{Dominance relative des taxons}

La valeur moyenne de la surface terrière dans la végétation d'arrière-plage est de $18,5 \mathrm{~m}^{2} /$ ha, pour les espèces dont la surface terrière est supérieure à $10 \mathrm{~cm}$. La famille des Ochnaceae domine avec une surface terrière de $7,31 \mathrm{~m}^{2} / \mathrm{ha}$. Lophira alata est alors l'espèce dominante de la ligne côtière, avec $39,62 \%$ du recouvrement total. Elle est suivie de Terminalia catappa (Combretaceae) qui occupe environ $3 \mathrm{~m}^{2} / \mathrm{ha}$ (soit $16,45 \%$ du recouvrement total).

\section{Menaces de la biodiversité}

Les activités humaines sont très fréquentes sur la ligne côtière de la réserve. Outre la présence de quelques petites parcelles de plantations ou d'anciennes plantations, toute la ligne côtière est constituée d'anciennes jachères et le lieu de fréquentation humaine permanente engendrant des conséquences énormes sur la biodiversité.

\section{Erosion côtière}

Le phénomène d'érosion de la côte est beaucoup plus prononcé et perceptible entre le campement de Youmè I et la pointe de Souellaba. La principale preuve de son existence est la présence d'arbres avec leurs racines à découvert. Ainsi 22 arbres de l'espèce Terminalia catappa ont été répertoriés comme menacés de chute par le phénomène d'érosion (Figure 5a). Par ailleurs, dans d'autres sites (Souelaba par exemple) ce sont les tiges des espèces rampantes qui sont systématiquement déracinées ou détruites par l'érosion (Figure $5 b)$. Les dunes sont aussi constamment remaniées par les eaux, et la végétation n'assure plus efficacement son rôle de protection du sol.

\section{Théorie de la transgression marine}

L'observation de la plage de Mombo permet de constater qu'il y a une progression de la ligne de plus haute marée, donc un recul du trait de côte. Sur une longueur de plage de $15 \mathrm{~km}$, la forêt a été immergée entrainant la mort des arbres sur pied (Figure 6), du fait 
de la modification de leur habitat originel.

Une couche épaisse de sable recouvre la terre sur une profondeur d'un mètre. Cette couche de sable stérile d'abord, sera recouverte d'une couche de litière qui va favoriser l'installation des espèces stolonifères et favorisera la germination du $T$. catappa et
Calollophyllym inophyllum. Cette observation concorde avec les témoignages de 30 habitants du village dont $80 \%$ affirment qu'il y a plusieurs dizaines d'années, les villages ont été engloutis dans l'océan. Les villages de Mombo, Eboule lombe, Mpêh auraient été déplacés plusieurs fois.

Tableau 1 : Distribution des espèces en fonction des familles.

\begin{tabular}{lllc}
\hline \multicolumn{1}{c}{ Familles } & Nombres d'espèces rencontrées & \multicolumn{1}{c}{ Familles } & $\begin{array}{c}\text { Nombres d'espèces } \\
\text { rencontrées }\end{array}$ \\
\hline Amaranthaceae & 1 & Erythroxylaceae & 1 \\
Annonaceae & 4 & Fabaceae & 8 \\
Anthericaceae & 1 & Flacourtiaceae & 1 \\
Apocynaceae & 1 & Irvingiaceae & 2 \\
Araceae & 1 & Ixonanthaceae & 1 \\
Arecaceae & 4 & Lamiaceae & 1 \\
Asclepiadaceae & 1 & Lauraceae & 1 \\
Asteraceae & 1 & Loganiaceae & 1 \\
Burseraceae & 1 & Malvaceae & 1 \\
Caesalpiniaceae & 2 & Marantaceae & 1 \\
Cecropiaceae & 1 & Melastomataceae & 2 \\
Celastraceae & 3 & Meliaceae & 1 \\
Clusiaceae & 1 & Mimosaceae & 2 \\
Combretaceae & 2 & Ochnaceae & 1 \\
Commelinaceae & 2 & Olacaceae & 1 \\
Connaraceae & 2 & Passifloraceae & 2 \\
Convolvulaceae & 5 & Piperaceae & 3 \\
Costaceae & 2 & Poaceae & 4 \\
Cyperaceae & 1 & Rhamnaceae & 1 \\
Dilleniaceae & 2 & Rubiaceae & 9 \\
Dioscoreaceae & 2 & Rutaceae & 1 \\
Dryopteridaceae & 1 & Ulmaceae & 1 \\
Ebenaceae & 2 & Violaceae & 1 \\
Euphorbiaceae & 4 & Vitaceae & 2 \\
Euphorbiaceae & 1 & Zingiberaceae & 1 \\
\hline
\end{tabular}

Tableau 2 : Liste des espèces des côtes de la Reserve de Faune de Douala-Edéa.

\begin{tabular}{ll}
\hline Familles & Noms scientifiques \\
\hline Amaranthaceae & Notosaerva brachiata \\
Annonaceae & Cleistopholis patens (Benth.) Engler \& Diels \\
& Pachypodanthium confine Engl. \& Diels \\
& Xylopia rubescens Oliv. \\
& Xylopia aethiopica (Dunal) A. Rich. \\
Anthericaceae & Crinum natans Baker \\
Apocynaceae & Landolphia sp. \\
Araceae & Anchomanes difformis (Blume) Engl. \\
Arecaceae & Calamus rotang L. \\
& Cocos nucifera $\mathrm{L}$.
\end{tabular}




\begin{tabular}{|c|c|}
\hline & $\begin{array}{l}\text { Elaeis guineensis Jacq. } \\
\text { Eremospatha } \mathrm{sp.}\end{array}$ \\
\hline Asclepiadaceae & Tylophora sp. \\
\hline Asteraceae & Chromolaena odorata (L.) R. M. King \& H. Rob. \\
\hline Burseraceae & Santiria trimera (Oliv.) Aubrév. \\
\hline & Anthonotha macrophylla P. Beauv. \\
\hline Caesalpiniaceae & Caesalpinia bonduc L. Roxb. \\
\hline Cecropiaceae & Musanga cecropioides R. Br. \& Tedlie \\
\hline Celastraceae & Maytenus sp. \\
\hline & $\begin{array}{l}\text { Salacia erecta }(\mathrm{G} . \text { Don) Walp. } \\
\text { S. pallescens Oliv. }\end{array}$ \\
\hline Clusiaceae & Calophyllum inophyllum L. \\
\hline Combretaceae & $\begin{array}{l}\text { Combretum platypterum (Welw.) Hutch. \& Dalziel } \\
\text { Terminalia catappa L. }\end{array}$ \\
\hline Commelinaceae & $\begin{array}{l}\text { Aneilema } \mathrm{sp} . \\
\text { Commelina } \mathrm{sp} .\end{array}$ \\
\hline Connaraceae & $\begin{array}{l}\text { Agelaea sp. } \\
\text { Cnestis ferruginea D. C. }\end{array}$ \\
\hline Convolvulaceae & $\begin{array}{l}\text { Ipomoea carica } \text { Sweet } \\
\text { Ipomoea involucatra } \mathrm{P} . \text { Beauv. } \\
\text { Ipomoea mauritiana Jacq. } \\
\text { Ipomoea pes-caprae (L.) R. Br. } \\
\text { I. sp. }\end{array}$ \\
\hline Costaceae & $\begin{array}{l}\text { Costus afer Ker-Gawl. } \\
\text { Costus tappenbeckianus J. Braun \& K. Schumann }\end{array}$ \\
\hline Cyperaceae & Scleria boivinii Stend. \\
\hline Dilleniaceae & $\begin{array}{l}\text { Tetracera alnifolia Wild. } \\
\text { Tetracera podotricha Gilg. }\end{array}$ \\
\hline Dioscoreaceae & $\begin{array}{l}\text { Dioscorea munitiflora Engl. } \\
\text { Dioscorea } \mathrm{sp} .\end{array}$ \\
\hline Dryopteridaceae & Nephrolepis biserrata (Sw.) Schott \\
\hline Ebenaceae & $\begin{array}{l}\text { Diospyros hoyleana } \mathrm{F} \text {. White } \\
\text { Diospyros } \mathrm{sp} .\end{array}$ \\
\hline Euphorbiaceae & $\begin{array}{l}\text { Alchornea cordifolia (Schumach. \& Thonn.) Müll. Arg. } \\
\text { Alchornea } \mathrm{sp.} \\
\text { Jatropha curcas L. } \\
\text { Manihot esculenta } \text { Crantz }\end{array}$ \\
\hline Euphorbiaceae & Uapaca sp. \\
\hline Erythroxylaceae & Erythroxylum mannii Oliv. \\
\hline Fabaceae & $\begin{array}{l}\text { Canavalia rosea (Sw.) D. C. } \\
\text { Centrosema pubescens Benth. } \\
\text { Dalbergia ecastaphyllum (L.) Taubert } \\
\text { Dalbergia sp. } \\
\text { Desmodium adscendens (Swartz) D. C. } \\
\text { Milletia sanagana Harms }\end{array}$ \\
\hline & $\begin{array}{l}\text { Milletia sp. } \\
\text { Vigna gracilis (Guill. \& Perr.) Hook. f. }\end{array}$ \\
\hline Flacourtiaceae & Homalium sp. \\
\hline Irvingiaceae & $\begin{array}{l}\text { Desbordesia glaucescens (Engl.) Van Tiegh. } \\
\text { Klainedoxa gabonensis Pierre ex Engl. }\end{array}$ \\
\hline Ixonanthaceae & Ochtocosmus calothyrsus Hutchinson \& Dalziel \\
\hline Lamiaceae & Clerodendron sp. \\
\hline Lauraceae & Beilschmiedia sp. \\
\hline Loganiaceae & Anthocleista schweinfhurthii Gilg. \\
\hline
\end{tabular}




\begin{tabular}{|c|c|}
\hline Malvaceae & Hibiscus mutabilis L. \\
\hline Marantaceae & Sarcophrynium brachystachium K. Schum. \\
\hline Melastomataceae & Dissotis sp. \\
\hline & Tristemma sp. \\
\hline Meliaceae & Carapa grandifolia Sprague \\
\hline Mimosaceae & $\begin{array}{l}\text { Albizia adianthifolia (Shumach.) W. F. Wight } \\
\text { Entada gigas (L.) Fawcett \& Rendle }\end{array}$ \\
\hline Ochnaceae & Lophira alata Banks ex C. F. Gaernt. \\
\hline Olacaceae & Ximenia americana $\mathrm{L}$. \\
\hline Passifloraceae & $\begin{array}{l}\text { Adenia sp. } \\
\text { Barteria nigritana Hook. f. }\end{array}$ \\
\hline Piperaceae & $\begin{array}{l}\text { Piper capense L. f. } \\
\text { Piper guineense Schumach. \& Thonn. } \\
\text { Piper sp. cf. guineense } \\
\text { Digitaria sp. }\end{array}$ \\
\hline Poaceae & $\begin{array}{l}\text { Echinochloa sp. } \\
\text { Olyra latifolia L. } \\
\text { Zea mays L. }\end{array}$ \\
\hline Rhamnaceae & $\begin{array}{l}\text { Maesopsis eminii Engler } \\
\text { Bertiera sp. }\end{array}$ \\
\hline Rubiaceae & $\begin{array}{l}\text { Gaertnera paniculata Benth. } \\
\text { Geophylla sp. } \\
\text { Morinda sp. } \\
\text { Oxyanthus unilocularis Hiern. } \\
\text { Psychotria sp. } \\
\text { Spermacoce princeae (K. Schum.) Verdc. } \\
\text { Tricalysia sp. }\end{array}$ \\
\hline Rutaceae & Clausena anisata (Wild.) Hook. f. ex Benth. \\
\hline Ulmaceae & Trema orientalis (L.) Bl. \\
\hline Violaceae & Rinorea sp. \\
\hline Vitaceae & $\begin{array}{l}\text { Ampelocissus sp. } \\
\text { Cissus sp. }\end{array}$ \\
\hline Zingiberaceae & Aframomum sp. \\
\hline
\end{tabular}

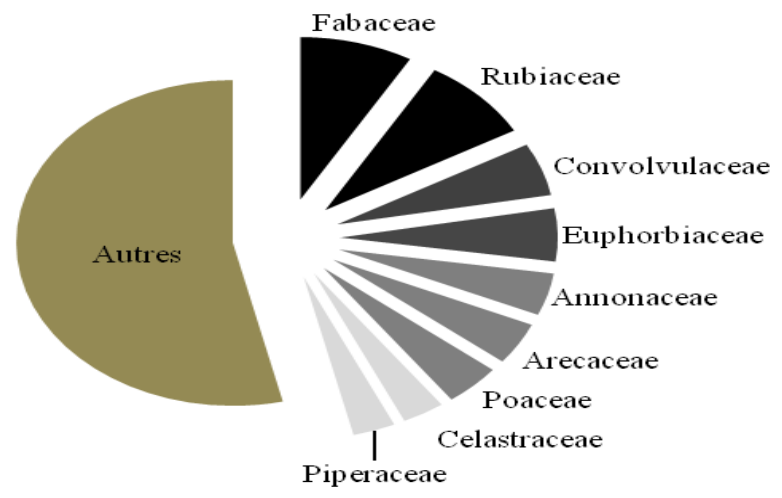

Figure 2 : Proportions des familles prépondérantes. 


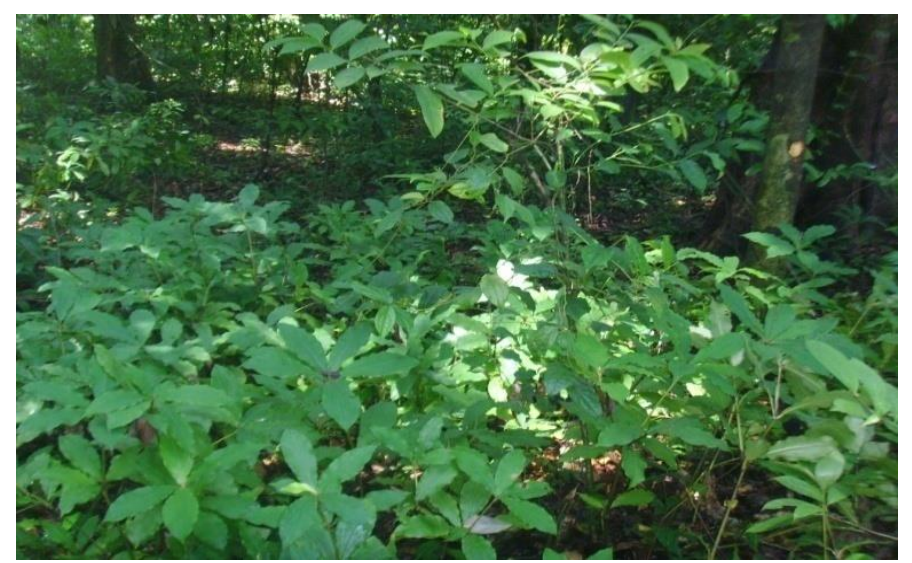

Figure 3 : Sous-bois à Costus tappenbeckianus (Costaceae).

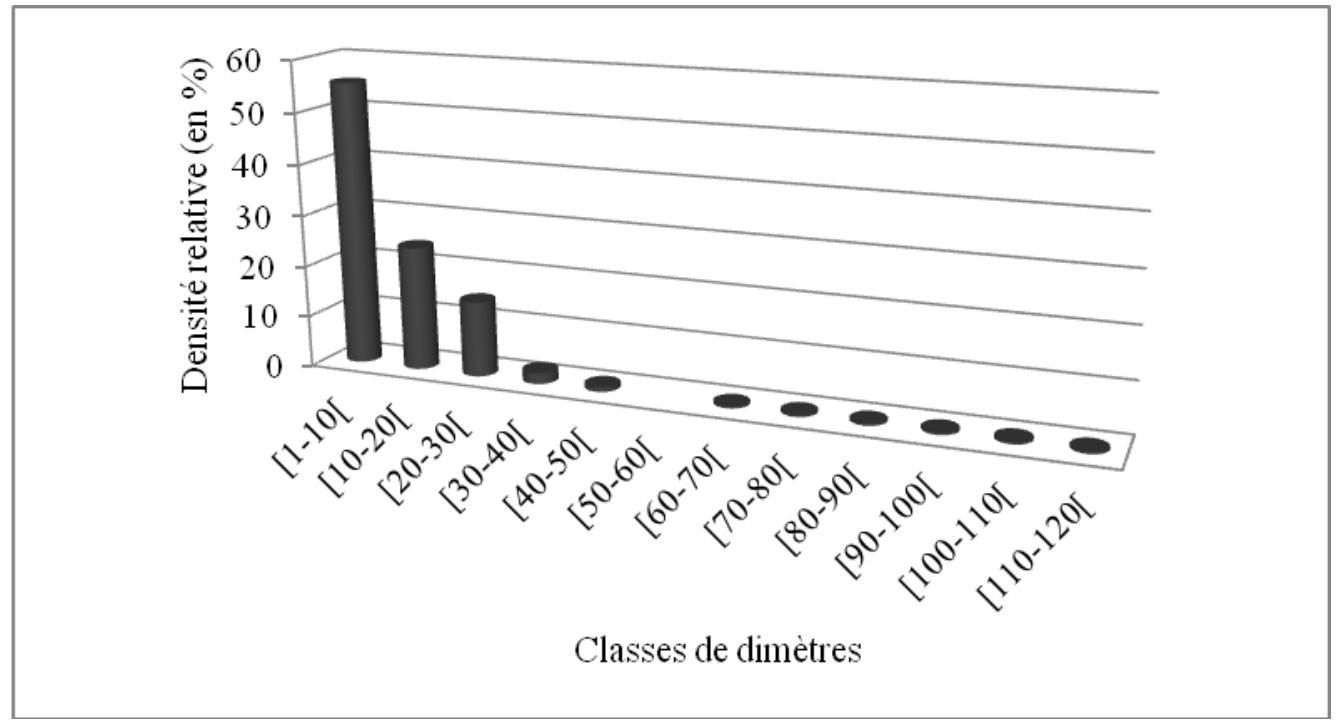

Figure 4: Distribution des individus dans les différentes classes de diamètres.

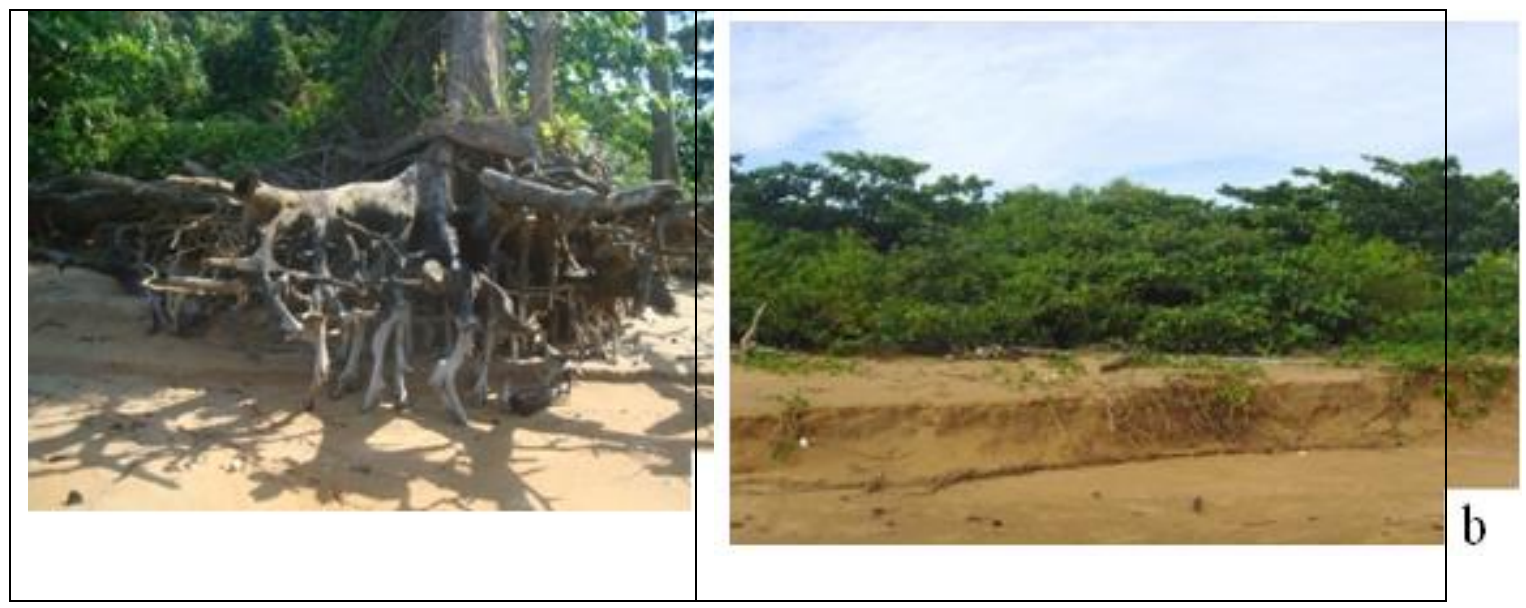

Figure 5 : Effets de l'érosion littorale. (a) arbre déraciné (Youmè I), (b) dune érodée (Moukoukè). 


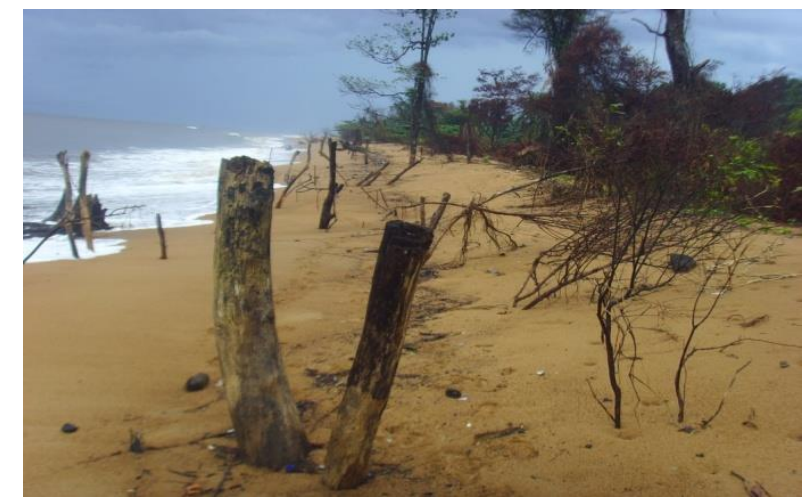

Figure 6 : Conséquences des activités anthropiques. Composition floristique, structure et menaces de la végétation de la ligne côtière de la Réserve de Faune de Douala-Edéa.

\section{DISCUSSION}

Les résultats obtenus présentent une richesse spécifique s'élève à 103 espèces recensées sur la ligne côtière de la RFDE. Les régions littorales ont une richesse spécifique faible comparées aux formations végétales de l'intérieur des terres. Cela est justifié par le fait que les plaines côtières, étant soumises à de fortes contraintes écologiques (milieu salé, sols sableux pauvres en éléments nutritifs et facilement inondables, etc.), voient leur nombre d'espèces dominantes restreintes (De Granville, 1990 ; Dauby, 2007).

Sur les plages de sable, la famille dominante sur le plan spécifique est celle des Fabacées. La dominance de cette famille n'est pas propre aux plages de la RFDE (Abuodha et al., 2003 ; Kouassi et al., 2010). L'espèce Ipomoea pes-caprae, fréquemment rencontrée elle aussi, et le genre Canavalia, sont des taxons pantropicaux et vraiment liés à la frange côtière (Cremers, 1985).

Un indice de Shannon (ISH) élevé correspond à des conditions de milieu favorables à l'installation de nombreuses espèces représentées par un petit nombre d'individus (Dajoz, 1982). La végétation de la ligne côtière de la RFDE présente un ISH de 3,56 bits. Celui-ci est relativement faible comparé à ceux obtenus dans d'autres milieux forestiers (Fongnzossié et al., 2008 ; Dongmo, 2006). Il apparait donc que la forêt juste à proximité de l'océan est très peu diversifiée. L'indice d'équitabilité de Piélou (Eq) vaut 0,49 . Les écosystèmes ayant atteint un niveau de maturité ont un Eq élevé entre 0,6 et 0,8 (Sonké, 1998). La valeur obtenue ici signifie que la majeure partie des individus inventoriés appartiennent à quelques espèces seulement. En effet, quatre espèces détiennent à elles seules plus de la moitié des effectifs. Calophyllum inophyllum et Terminalia catappa, particulièrement, sont abondantes sur les plages et arrières-plages de la réserve grâce à leur adaptation aux milieux sableux et leur grande tolérance aux embruns salés (Angoni, 2005).

S'agissant de la distribution diamétrique, les individus de diamètre inférieur à $10 \mathrm{~cm}$ regroupent plus de $55 \%$ du total des individus. Ce nombre élevé de petits diamètres et la distribution exponentielle en décroissance qui a été obtenue caractérisent une bonne régénération dans le sous-bois et une population s'entretenant elle-même (Fongnzossié et al., 2008).

S'agissant du recouvrement, il est de $18,5 \mathrm{~m}^{2} /$ ha pour les individus d'au moins $5 \mathrm{~cm}$ de diamètre. Les forêts de la bande côtière de l'UTO de Campo-Ma'an atteignent des valeurs de ST de $53 \mathrm{~m}^{2} /$ ha (Angoni, 2005). Celles sur alluvions en Nouvelle-Calédonie atteignent des valeurs de ST de $47 \mathrm{~m}^{2} / \mathrm{ha}$, pour les individus de diamètre supérieur à $10 \mathrm{~cm}$ 
(Jaffré et Veillon, 1991). La faible valeur de la ST de la forêt de la ligne côtière de la RFDE est due à la faible densité des individus d'une part, et d'autre part au très faible nombre d'arbres de gros diamètre. Les arbres dont le diamètre est compris entre 60 et $120 \mathrm{~cm}$ ne représentent que $2,6 \%$ de l'effectif total, mais constituent plus de la moitié de la surface terrière.

Selon Mosango (1990) et Sokpon (1995), la surface terrière est un bon outil d'appréciation de l'état de maturité des forêts. Elle est en moyenne comprise entre 30 et 40 $\mathrm{m}^{2} /$ ha pour les forêts matures. Au regard des résultats obtenus, il convient d'affirmer que les peuplements explorés ne sont pas matures, ce qui conforte les affirmations de Letouzey (1985) sur la végétation jeune de la ligne côtière de la réserve, en constante renouvellement à cause du mouvement de va et viens de la marrée.

Le phénomène d'élévation du niveau de la mer a été constaté sur les plages de sable du secteur Sud de la réserve (Angoni, 2011) notait déjà une augmentation de la vitesse d'élévation du niveau moyen de la mer avec la chute des arbres dans la zone de Mombo. Selon GIEC (2007), une élévation du niveau moyen de la mer de $59 \mathrm{~cm}$ est plausible à l'horizon 2100. Au Congo, un village a déjà été entièrement englouti (Sitou, 2007). L'érosion de la côte est aussi perceptible sur les côtes de la réserve, comme sur d'autres côtes du pays. En effet, Yowou Nzofang (2009) trouve que 5,3\% de la côte entre les embouchures de la Lobé et du Ntem sont érodés. Outre les causes anthropiques, l'élévation du niveau de la mer aggrave également le phénomène d'érosion des systèmes sableux. Anonyme (2007) le précise aussi : une élévation du niveau marin devrait avoir des effets néfastes sur les systèmes naturels, comme l'accroissement des risques auxquels sont exposées les côtes, notamment en matière d'érosion.

\section{Conclusion}

La zone côtière de la Réserve de Faune de Douala-Edéa se compose de trois types d'habitats : les mangroves et la végétation des plages de sable ou cordons littoraux et les zones marécageuses. $\mathrm{La}$ composition spécifique et la diversité des familles restent faibles par rapport aux autres côtes atlantiques à cause d'une action anthropique très poussée le long des plages. On identifie les espèces ligneuses telles que: Terminalia catappa, Callophyllum inophyllum, Lophira alata, Ximeria americana, Anthocleista schweinfurthii, Barteria nigritana, Uapaca heudelotii et les herbacées Canavalia rosa, Rimerea maritima, Ipomoea spp. Ces espèces caractéristiques restent communes à l'ensemble de la côte, quoique certaines présentent une répartition pantropicale.

La répartition des arbres en fonction des classes de diamètre, montre une forte fréquence des tiges ayant un diamètre inférieur à $10 \mathrm{~cm}$. Les arbres à gros diamètre se raréfient. Cette présence des tiges à petit diamètre influence le recouvrement végétal dominé par les arbres dont le diamètre est inférieur à $10 \mathrm{~cm}$.

Malgré la relative pauvreté spécifique et la faible diversité floristique de la ligne côtière de la RFDE, les activités anthropiques entrainent des coupes incontrôlées de bois laissant en places des jeunes arbres qui colonisent la côte.

\section{REFERENCES}

Abuodha JOZ, Musila WM, Van der Hagen H. 2003. Floristic composition and vegetation ecology of the Malindi Bay coastal dune field, Kenya. Journal of Coastal Conservation, 9(2): 97-112.

Angoni H. 2005. Biologie et écologie des tortues marines en rapport avec les écosystèmes côtiers. Conservation et aménagement. Thèse de doctorat $3^{\text {ème }}$ cycle, Université de Yaoundé I. 125 p.

Angoni. 2011. Conservation des tortues marines et amélioration de la qualité de 
vie dans les villages côtiers riverains de l'UTO Campo-Ma'an et dans la Réserve de Faune de Douala-Edéa. Rapport du Projet GEF/UNDP/SGP. 31 p.

Anonyme. 1992. Convention sur la diversité biologique. Nations Unies, Rio De Janeiro. $30 \mathrm{p}$.

Anonyme. 2001. Incidences de l'évolution du climat dans les régions : évaluation de la vulnérabilité. Chapitre 2: Afrique. Rapport spécial du Groupe de travail II du GIEC. 53 p.

Anonyme. 2006. Rapport du niveau d'avancement des activités liées au processus de reclassement de la réserve de faune de douala-Edéa en parc national. 28 p.

Anonyme. 2007. Bilan 2007 des changements climatiques. Contribution des Groupes de travail I, II et III au quatrième Rapport d'évaluation du Groupe d'experts intergouvernemental sur l'évolution du climat. GIEC, Genève, Suisse, 73 p + annexes.

Braun-Blanquet J. 1932. Plant Sociology. Translated by Fuller GD and Conard HS. McGraw p-Hill Book Co., Inc.: New York and London; 439 p.

Cremers G. 1985. Végétation et flore illustrée des bords de mer: exemple de l'île de la Cayenne. La Nature et l'Homme en Guyane. Cayenne, ORSTOM ; 65-75.

Dajoz R. 1982. Précis d'Ecologie (4 ${ }^{\mathrm{ème}}$ édition). Bordas : Paris ; $503 \mathrm{p}$.

Dauby G. 2007. Étude floristique et biogéographique du Parc national de la Pongara (Gabon). Mémoire de DEA, Université Libre de Bruxelles ; 87 p.

De Granville J-J. 1990. Les formations végétales actuelles des zones côtières et subcôtières des Guyanes. Colloque : l'évolution des littoraux des Guyanes et de la zone Caraïbe méridionale pendant le Quaternaire. P.I.C.G. 274: 9-14, Novembre 1990, Cayenne.

De Kam M, Fines JP, Akogo MG. 2002. Schéma directeur pour le développement de l'Unité Technique Opérationnelle de Campo-Ma'an, Cameroun. Tropenbos International, Kribi-Cameroun ; 87 p.

Dongmo MD. 2006. Etudes floristiques et ethnobotaniques dans un village de la zone forestière du Cameroun: cas de Nkolbibanda. Mémoire de DEA, Université de Yaoundé I. 47 p + annexes.

Fongnzossie F E, Tsabang N, Nkongmeneck BA, Nguenang GM, Auzel P, Christina E, Kamou E, Balouma JM, Apalo P, Halford M, Valbuena M, Valère M. 2008. Les peuplements d'arbres du Sanctuaire à gorilles de Mengamé au sud Cameroun. Tropical Conservation Science, 1(3): 204221.

Husch B, Beers WT, Kershaw AJ. 2003. Forest Mensuration $\left(4^{\text {th }}\right.$ Edn). Wiley.

Jaffré T, Veillon J-M. 1991. Étude floristique et structurale de deux forêts denses humides sur roches ultrabasiques en Nouvelle-Calédonie. Bull. Mus. natn., Paris $4^{\text {ème }}$ sér., 12, 1990 (publ. 1991), section B Adansonia ${ }^{\text {os }}$ 3-4 : 243-273.

Kimpouni V, Loumeto JJ, Mizingou J. 2008. Diversité floristique du peuplement ligneux de la forêt monodominante à Okoumé (Aucoumea klaineana P.) du littoral congolais. Annales de l'Université Marien Ngouabi, 2008 : 9-21.

Kouassi A, Yao CYA, Ipou J, Kamanzi K. 2010. Diversité floristique des zones côtières pâturées de la Côte d'Ivoire : cas $\mathrm{du}$ cordon littoral Port-Bouët-GrandBassam (Abidjan). Sciences et Nature, 7(2) : 69-86.

Letouzey R. 1985. Notice de la carte phytogéographique du Cameroun au 1:500000: Institut de la carte internationale de la végétation. ToulouseFrance; pp 95-142.

Mosongo M. 1990. Contribution à l'étude botanique et biogéographique de l'écosystème forêt en forêt équatoriale (Ile Kolongo, Zaïre). Thèse de Doctorat. Université Libre de Bruxelles ; 446 p. 
Nanko GL, Angoni H, Motto Mallo JG, Nkoum L, Ekwelgen C, Same Ekobo, Abega R. 2010. Etude d'Impact Environnemental et Social (EIES) du Développement du champ de MVIA-1. Rapport Ere Développement; 303 p.

Piélou EC. 1965. Species diversity and pattern diversity in study of ecological succession. J. Theor. Biol., 10: 370-383.

Shannon CE. 1948. A mathematical theory of communications. Bell System Technical Journal, 27: 379-423.

Sitou L. 2007. Dynamique et interprétation de l'érosion côtière dans la région de PointeNoire. Annales de l'Université Marien Ngouabi, 8(1) : 44-56.

Sokpon N. 1995. Recherche écologique sur la forêt dense semi-décidue de Pobè au Sudest du Bénin: groupements végétaux, structure, régénération naturelle et chute de litière. Thèse de Doctorat, Université Libre de Bruxelles ; $350 \mathrm{p}$.

Sonke B. 1998. Études floristiques et structurales des forêts de la Réserve de faune du Dja (Cameroun). Thèse de doctorat, Université Libre de Bruxelles; $266 \mathrm{p}$.

Vinchon C, Oliveros C, Pedreros R, Lenotre N. 2006. Response of the Coastline to Climate Change. Specific Report for the RESPONSE Project LIFE-Environment.

Vivien J, Faure J-J. 1985. Arbres des Forêts Denses d'Afrique Centrale. Agence de Coopération Culturelle et Technique ; 5063.

Youwou. 2009. Impact des activités anthropiques sur la dynamique côtière de l'Unité Technique Opérationnelle (UTO) Campo Ma'an. Mémoire d'étude, Université de Yaoundé I; 62p. 